\title{
RADICAL AND SEMISIMPLE CLASSES WITH SPECIFIED PROPERTIES
}

\author{
W. G. LEAVITT
}

\begin{abstract}
Conditions are given on a radical (semisimple) property to ensure the existence of a construction for the smallest radical (semisimple) class with the property, containing a given class of rings. This generalizes earlier results on smallest hereditary or strongly hereditary radical classes, and hereditary semisimple classes. In the last section certain classes of rings are shown to admit a construction for the largest radical (semisimple) class contained in the given class. This leads to theorems on largest radical (semisimple) classes dual to the already established smallest theorems.
\end{abstract}

1. Introduction. Let $w$ be an arbitrary universal class of (not necessarily associative) rings. For a subclass $\Re \subseteq W$ a subclass $\mathcal{P} \supseteq \Re($ with a specified property is said to be a smallest class containing $\mathfrak{N}$ with the property if $P \subseteq \mathfrak{F C}$ for all $\mathfrak{H} \supseteq \mathfrak{N}$ with the property. The hereditary radical and strongly hereditary radical [1] are examples of properties admitting smallest classes for any subclass $\mathfrak{T}$ of an arbitrary universal class. We will give a sufficient condition on a radical property that it admit smallest classes (Theorem 1). The proof yields, in fact, a construction (for an arbitrary $\mathfrak{T}$ ) of the smallest radical with the specified property. Theorem 2 gives an analogous construction for certain smallest semisimple classes. A special case is the result [2, Theorem 2] for hereditary semisimple classes.

In the last section is considered the dual problem of largest subclass, namely, for a given class $\mathfrak{T}$, a subclass of $\mathfrak{T}$ with a specified property and containing all subclasses of $\mathfrak{T}$ with the property. Certain classes are shown to admit constructions for largest radical or semisimple classes. This construction also yields an easy way of constructing the "upper hereditary radical" of Rjabuhin [3]. It is then found possible to "dualize" in such a way that (with suitable restrictions) the concept of largest radical class is dual to smallest semisimple class. Similarly largest semisimple and smallest radical become dual.

In the constructions we use a number of class functions. These

Received by the editors May 5, 1969 and, in revised form, September 11, 1969. A MS Subject Classifications. Primary 1630, 1644; Secondary 1710.

Key Words and Phrases. General radical classes, semisimple classes, hereditary radical, smallest class of rings with a specified property, largest class of rings with a specified property contained in a given class, lower radical construction. 
include the lower radical $\operatorname{LN}[4$, p. 13$]$ which is the smallest radical class containing $\mathfrak{T}$, and the hereditary closure $9 \mathfrak{T}[\mathbf{5}, \mathrm{p} .1114]$ which is the smallest hereditary class containing $\Re$. Other useful functions are $\delta \mathfrak{T}=\{R \in W \mid$ for every $0 \neq I \triangleleft R$ there is some $0 \neq I / J \notin \mathfrak{M}\}$ (we use the notation $I \triangleleft R$ to mean $I$ is an ideal of $R$ ), and $\mathcal{r}$, $=\{R \in w \mid$ for every $0 \neq R / I$ there is some $0 \neq J \triangleleft R / I$ with $J \notin \Re\}$. It is well known that when $\mathscr{T}$ is radical $\$ \Re($ is its semisimple class, and when $\mathfrak{T}$ is semisimple its radical class is $น \mathfrak{T}$.

2. The smallest radical construction. Write $J$ for the class of all subclasses of $W$ and $R$ for the class of all radical subclasses of $W$. We will call a function $F: R \rightarrow J$ admissible if it has properties (i) $\odot \subseteq F \odot$ for all $\odot \in R$, (ii) if $\mathcal{P}_{1}, \odot_{2} \in R$ with $\mathcal{P}_{1} \subseteq \mathcal{P}_{2}$ then $F P_{1} \subseteq F P_{2}$, and (iii) if $\left\{\rho_{\alpha}\right\} \subseteq R$ defined for all ordinals $\alpha$ and such that $\alpha<\beta$ implies $\rho_{\alpha} \subseteq \mathcal{P}_{\beta}$, then for $\odot$ a radical class, $\odot=\bigcup_{\alpha} \mathcal{P}_{\alpha}$ implies $F \odot \subseteq \bigcup_{\alpha} F \odot_{\alpha}$.

Theorem 1. If $F$ is admissible and Th an arbitrary subclass of $W$, then there exists a smallest $\overline{\mathfrak{M}} \in R$ such that $\mathfrak{M} \subseteq \overline{\mathscr{M}}$ and $\overline{F \mathscr{M}}=\overline{\mathfrak{M}}$.

Proof. Let $\mathfrak{T}_{1}=\mathscr{L} \mathscr{N}$ and if $\beta$ is a nonlimit ordinal define $\mathfrak{T}_{\beta}$ $=\mathscr{L} F \mathfrak{N}_{\beta-1}$. For $\beta$ a limit ordinal let $\mathfrak{T}_{\beta}=\mathfrak{L} \bigcup_{\alpha<\beta} \mathfrak{T}_{\alpha}$, and write $\overline{\mathfrak{N}}=U \mathfrak{M T}_{\beta}$ taken over all ordinals $\beta$. We know by (i) that $\mathfrak{T M}_{\beta-1}$ $\subseteq F \mathbb{M}_{\beta-1} \subseteq \mathfrak{M T}_{\beta}$, so $\alpha<\beta$ implies $\mathfrak{M T}_{\alpha} \subseteq \mathfrak{M T}_{\beta}$ for all ordinals $\alpha, \beta$. Also $\overline{\mathfrak{N}}$ is a union of radical classes and so is homomorphically closed. To show $\overline{\mathfrak{T}}$ is a radical class we thus need only show (see e.g. $[4, \mathrm{p} .4]$ ) that it has property

(A). If $R \in W$ is such that for all $0 \neq R / I$ there is some $0 \neq J \triangleleft R / I$ with $J \in \overline{\mathscr{N}}$, then $R \in \overline{\mathfrak{N}}$.

But then $J \in \mathscr{I T}_{\alpha}$ for some $\alpha$ and since the collection of all such $J$ is a set there must be a largest $\alpha$, say $\gamma$. Thus all $J \in \mathscr{T}_{\gamma}$ and since $\mathfrak{N}_{\gamma}$ is a radical class this implies $R \in \mathfrak{M}_{\gamma} \subseteq \overline{\mathscr{N}}$. Thus $\overline{\mathfrak{T}} \in R$ and by (i), $\overline{\mathfrak{N}} \subseteq F \overline{\mathscr{T}}$. But $F \overline{\mathscr{M}} \subseteq \bigcup_{\beta} F \mathscr{N}_{\beta}$ by (iii) and since $F \mathscr{N}_{\beta} \subseteq \mathscr{M T}_{\beta+1}$ it follows that $F \overline{\mathscr{M}} \subseteq \overline{\mathfrak{N}}$. Thus $F \overline{\mathscr{M}}=\overline{\mathscr{M}}$. Finally suppose $\mathfrak{T} \subseteq \odot$ where $P \in R$ and $F \odot=\odot$. Since $\mathscr{L} \mathscr{T}$ is the smallest radical containing $\mathscr{T}$, we have $\mathfrak{M}_{1} \subseteq \beta$. Thus suppose for induction that $\mathfrak{N}_{\alpha} \subseteq \beta$ for all $\alpha<\beta$. Then $\mathcal{L U}_{\alpha<\beta} \mathfrak{T}_{\alpha} \subseteq \mathscr{L} \mathcal{P}=\beta$ when $\beta$ is a limit ordinal. Otherwise $\mathfrak{T}_{\beta-1} \subseteq \mathcal{P}$ whence $F \mathfrak{M}_{\beta-1} \subseteq F \mathcal{P}=\mathcal{P}$ and so $\mathfrak{N}_{\beta} \subseteq \mathcal{L} \mathcal{P}=\mathcal{P}$. Thus by induction $\overline{\mathfrak{M}} \subseteq \odot$.

CoROllary 1. Every class $\mathfrak{M} \subseteq W$ is contained in a smallest hereditary radical class and a smallest strongly hereditary class.

Proof. This follows from the admissibility of both $g$ and $\mathscr{g} \mathcal{G}$, where $G$ is defined as in $[1,(2)$, p. 704]. 
3. The smallest semisimple construction. In [2] a class $\mathfrak{M}^{\prime} \supseteq \mathfrak{N}$ was called an $s$-completion of $\mathfrak{T}$ if it has the property

(B). If $R \in \mathfrak{M}^{\prime}$ then for every $0 \neq I \triangleleft R$ there exists some $0 \neq I / J \in \mathfrak{N}^{\prime}$. It is well known that $\mathfrak{T}^{\prime}$ is semisimple if $\mathfrak{T}^{\prime}$ also satisfies

(C). For any $R \in W$, if for every $0 \neq I \triangleleft R$, there exists some $0 \neq I / J \in \mathfrak{M}^{\prime}$, then $R \in \mathfrak{M}^{\prime}$ (see e.g. [4, p. 5]). It is also well known $\left[4\right.$, p. 7] that if $\mathfrak{T}^{\prime}$ satisfies (B) then $\mathcal{U}^{\prime} \boldsymbol{\prime}^{\prime}$ is a radical class such that $\mathfrak{N}^{\prime} \subseteq \delta \mathcal{S} \mathfrak{T}^{\prime}$ with $\delta \mathcal{M} \boldsymbol{I}^{\prime}$ the smallest semisimple class containing $\mathfrak{N}^{\prime}$. We will call a function $F: \Im \rightarrow J s$-admissible if it has the properties (i) $F \mathfrak{M}$ is an $s$-completion of $\mathscr{M}$ for all $\mathfrak{M} \in \mathfrak{J}$, (ii) if $\mathfrak{M} \subseteq \mathfrak{N}$ then $F \mathfrak{M} \subseteq F \Re$, and (iii) if $\mathcal{Y}$ is the class of all semisimple subclasses of $\mathscr{W}$ and $\left\{\mathfrak{M}_{\alpha}, \cup \mathscr{T}_{\alpha}\right\} \subseteq \mathcal{Y}$, where $\alpha<\beta$ implies $\mathscr{M}_{\alpha} \subseteq \mathfrak{M}_{\beta}$, then $F\left(U \mathscr{T}_{\alpha}\right)$ $\subseteq U F\left(\mathscr{T}_{\alpha}\right)$.

Theorem 2. Let $F$ be an s-admissible function. Then for all $\mathfrak{T} \in \mathfrak{J}$ there exists a smallest $\overline{\mathfrak{M}} \in \mathcal{Y}$ such that $\mathfrak{\Re} \subseteq \overline{\mathfrak{N}}$ and $F \overline{\mathrm{N}}=\overline{\mathfrak{N}}$.

Proof. Define $\mathfrak{T}_{1}=\delta \mathcal{U} F \mathfrak{T}$ and for $\beta$ a nonlimit ordinal define

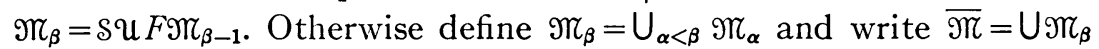
taken over all ordinals $\beta$. Clearly $\alpha<\beta$ implies $\mathfrak{M}_{\alpha} \subseteq \mathfrak{T}_{\beta}$, and since $\overline{\mathfrak{M}}$ is the union of semisimple classes it has property (B). We thus have $\overline{\mathfrak{N}} \subseteq \delta น \bar{\Re}$. But if $R \in \delta u \bar{\Re}$ then for every $0 \neq I \triangleleft R$ there is some $0 \neq I / J \in \overline{\mathscr{T}}$. Thus $I / J \in \mathscr{M}_{\alpha}$ for some ordinal $\alpha$, and so for $\gamma$ the largest such ordinal we have all such $I / J \in \mathfrak{M}_{\gamma}$. Since $\mathfrak{M}_{\gamma} \subseteq \mathfrak{M}_{\gamma+1}$ and $\mathfrak{M}_{\gamma+1}$ is semisimple, it follows from (C) that $R \in \mathfrak{M}_{\gamma+1} \subseteq \mathfrak{M}$. Accordingly $\overline{\mathscr{M}}=\delta u \overline{\mathscr{M}}$ is semisimple. Now $\overline{\mathscr{M}} \subseteq F \overline{\mathscr{M}}$ and the reverse inequality follows from (iii). Finally it is clear by induction that if $\mathfrak{N} \subseteq \mathscr{N}$ where $\mathscr{N}$ is a semisimple class for which $F \mathfrak{N}=\mathfrak{N}$ then $\overline{\mathfrak{N}} \subseteq \mathfrak{N}$.

Since $g$ is $s$-admissible we have

COROLlaRY 2. Every class $\mathfrak{M} \subseteq W$ is contained in a smallest hereditary semisimple class.

4. Largest radical and semisimple classes. In this section we will consider the dual problem of largest radical or semisimple classes with given properties. This discussion will be based on the following construction of a largest radical class, and an analogous construction (Theorem 4) of a largest semisimple class.

THEOREM 3. If $\mathfrak{T}$ is a class containing a largest class $\mathfrak{M}_{1}$ satisfying condition (A) then there exists a largest $\rho \in R$ such that $\rho \subseteq \mathfrak{T}$ and $a$ largest hereditary radical class $\mathfrak{H} \subseteq \mathcal{P} \subseteq \mathfrak{M}$.

Proof. For each integer $n \geq 1$ define $\mathfrak{M}_{n+1}=\left\{R \in \mathfrak{M}_{n} \mid\right.$ if $0 \neq R / I$ then $0 \neq J \triangleleft R / I$ for some $\left.J \in \mathscr{N}_{n}\right\}$. Then set $\mathcal{P}=\bigcap_{\mathfrak{M}_{n}}$. For induction 
assume that $\mathfrak{T}_{k}$ has property (A) for all $k \leqq n$ and suppose $R \in W$ to be such that every $0 \neq R / I$ has a nonzero ideal in $\mathfrak{T}_{n+1}$. Since $\mathfrak{T}_{n+1}$ $\subseteq \mathfrak{M}_{n}$ these ideals are all in $\mathfrak{M}_{n}$ so by property (A) $R \in \mathfrak{M}_{n}$. But then by definition $R \in \mathfrak{M}_{n+1}$, and so $\mathfrak{M}_{n+1}$ has property (A). Thus by induction $P$ has property (A). Also if $\bar{R}$ is an image of an $R \in \mathbb{N}_{n}$ then any nonzero image of $\bar{R}$ has a nonzero ideal in $\mathfrak{T}_{n}$.

By property (A) $\bar{R} \in \mathscr{T}_{n}$ and so $R \in \mathcal{P}$ implies $\bar{R} \in \mathcal{P}$. Thus $P$ is homomorphically closed and so is a radical class.

Now if $\mathcal{P}^{\prime} \subseteq \mathscr{M}$ with $\boldsymbol{P}^{\prime} \in \mathbb{R}$ then $\mathcal{P}^{\prime}$ has property (A) and so $\mathcal{P}^{\prime} \subseteq \mathbb{M}_{1}$. Assume for induction that $\boldsymbol{P}^{\prime} \subseteq \mathfrak{M}_{n}$. If $R \in \mathcal{P}^{\prime}$ then $R \in \mathscr{M}_{n}$ and since $\rho^{\prime}$ is radical every $0 \neq R / I \in \mathcal{P}^{\prime} \subseteq \mathfrak{M}_{n}$. Thus $\boldsymbol{P}^{\prime} \subseteq \mathfrak{M}_{n+1}$ and so by induction $\mathcal{P}^{\prime} \subseteq \mathfrak{N}_{n}$ for all $n$. Therefore $\mathcal{P}^{\prime} \subseteq \mathcal{P}$ and so $\rho$ is the largest radical class contained in $\mathfrak{T}$.

To construct the largest hereditary radical let $\mathfrak{H}=\{R \in \mathcal{S} \mid \mathscr{g}\{R\} \subseteq \mathcal{P}\}$. Clearly $\mathcal{F}$ is hereditary. In fact it is the largest hereditary subclass

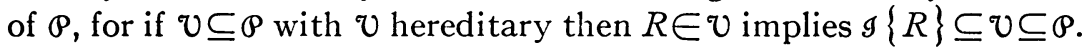
Thus $R \in \mathcal{H}$ and so $V \subseteq \mathfrak{H C}$. Now $\mathfrak{H} \subseteq \mathcal{P}$ so that $\mathfrak{H} \subseteq \mathcal{L} \mathcal{H} \subseteq \mathcal{P}$. But by [6] $\mathscr{L} \mathcal{H}$ is hereditary and so $\mathscr{L} \mathcal{H} \subseteq \mathcal{H}$. Thus $\mathcal{H}=\mathscr{L} \mathcal{H}$ and is the largest hereditary radical contained in $\rho$. But $\beta$ contains all radical subclasses of $\mathfrak{T C}$ so $\mathfrak{H C}$ is also the largest hereditary radical class contained in $\mathfrak{T}$.

Remark that in [3] Ju. M. Rjabuhin gave a construction (valid only for associative or alternative rings) for the "upper hereditary radical" which is the largest hereditary radical class contained in a class satisfying condition (A). The Rjabuhin result is thus a special case of the above theorem. Our construction is also considerably simpler than that of [3]. Also in this paper, certain classes $\Re_{\alpha}$ are defined (for all ordinals $\alpha$ ) whose union is the "lower hereditary radical." However this is just the smallest hereditary radical class containing a given class $\mathfrak{M}$, that is, by [6], LIM.

Note that it is quite possible for $P$ (or $\mathfrak{H}$ ) to be zero:

EXAMPLE 1. Let $W$ be an arbitrary universal class containing $J \oplus K$, where $J$ and $K$ are nonisomorphic simple rings with unit. Then the class $\Re=\{0, J \oplus K\}$ has property (A), since if $J \oplus K \triangleleft R$ then $J \oplus K$ is a direct summand of $R$. However 0 is the only radical class contained in $\mathfrak{T}$.

Also remark that Theorem 3 does not apply to all $\mathfrak{T C}$ since there exist classes not containing a largest (or even a maximal) radical or hereditary radical class. One such case is:

EXAmple 2. Let $w$ be any universal class large enough to contain an infinite set $\mathcal{V}$ of simple rings. It is well known that if $\mathscr{T}$ is homomorphically closed, a simple ring $R \in \mathscr{L N}$ if and only if $R \in \mathfrak{M}$. Thus 
if $a$ and $B$ are subsets of $v$ then $a \cap \mathcal{L} B \subseteq Q \cap B \subseteq \mathcal{L}(a \cap B)$. Since by [6] $\mathscr{L} B$ is hereditary, it is easy to see by induction that $a_{\alpha} \cap \mathscr{L} B$ $\subseteq \mathcal{L}(Q \cap B)$ for all ordinals $\alpha$. Thus $\mathcal{L} Q \cap \mathcal{L} B=\mathcal{L}(Q \cap B)$. Let $\left\{\Theta_{i}\right\}$ be the set of all finite subsets of $v$ and let $\mathscr{T C}=U \mathscr{L} B_{i}$. Suppose $\rho$ is a radical subclass of $\mathfrak{T}$. By what we just showed, there is for each $K \in P$ a unique minimal subset $B$ of $V$ such that $K \in \mathcal{L} \beta$. If the union of all such $B$ 's (for all $K \in P$ ) were infinite we would be able to choose a sequence $K_{i} \in \mathcal{P}(i=1,2, \cdots)$ such that $K_{i} \in \mathscr{L} \mathbb{B}_{i}$ and such that $\cup B_{i}$ is infinite. But $K=\sum \oplus K_{i} \in \mathcal{P} \subseteq \mathbb{M}$ and so $K \in \mathscr{L} B$ for some finite set $B$. Then all $K_{i} \in \mathcal{L} B$ and by the minimality of the $B_{i}$ we would get $B_{i} \subseteq B$. Thus the contradiction $\cup_{B_{i}} \subseteq B$. We conclude that

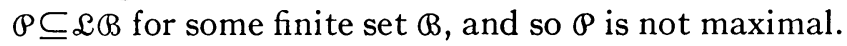

Note that there are classes satisfying Theorem 3 which do not themselves satisfy condition (A):

ExAmple 3. Using the same rings as in Example 1, the class $\{0, J, J \oplus K\}$ does not have property (A), since it does not contain $J \oplus J$, but does satisfy Theorem 3 .

By a somewhat analogous construction we obtain

Theorem 4. Let $\mathfrak{T} \in \mathfrak{J}$ contain a largest class $\mathfrak{T}_{1}$ satisfying condition (C). Then there exists a largest $Q \in \mathcal{Y}$ such that $Q \subseteq \mathscr{~} \subseteq$.

Proof. If $\beta \geqq 2$ is a nonlimit ordinal define $\mathfrak{T}_{\beta}=\left\{R \in \mathfrak{M T}_{\beta-1} \mid\right.$ for all $0 \neq I \triangleleft R$ there is some $\left.0 \neq I / J \in \mathfrak{T}_{\beta-1}\right\}$. Otherwise define $\mathfrak{N}_{\beta}=\bigcap_{\alpha<\beta} \mathfrak{T}_{\alpha}$, and write $Q=\bigcap \Re_{\beta}$ taken over all ordinals $\beta$.

Notice that the radical in Theorem 3 is obtained at the first transfinite ordinal (as $\cap \Re_{n}$ taken over all integers $n$ ), whereas here we may need to take the intersection over all ordinals.

Assume for induction that all $\mathfrak{M}_{\alpha}$ for $\alpha<\beta$ satisfy condition (C). Let $R \in W$ be such that every $0 \neq I \triangleleft R$ has an image $0 \neq I / J \in \mathbb{M}_{\beta}$. If $\beta-1$ exists, then since $\mathfrak{T}_{\beta} \subseteq \mathfrak{N}_{\beta-1}$ which has property (C), it follows that $R \in \mathscr{M}_{\beta-1}$. But then by definition $R \in \mathscr{M}_{\beta}$. If $\beta$ is a limit ordinal then $I / J \in \mathscr{N}_{\alpha}$ for all $\alpha<\beta$ so again by the induction hypothesis $R \in \mathscr{T}_{\alpha}$, and so $R \in \cap \mathfrak{T}_{\alpha}=\mathfrak{M}_{\beta}$. Thus $\mathfrak{T}_{\beta}$ has property (C) and the induction is complete. Clearly a similar proof shows that also $Q$ has property (C).

Now let $R \in Q$ and consider any $0 \neq I \triangleleft R$ for which all $0 \neq I / J \notin Q$. Since $\mathcal{Q}=\bigcap_{\mathfrak{T}_{\beta}}$, in particular $R \in \mathfrak{T}_{2}$ so $I$ has at least some $0 \neq I / J$ $\in \mathfrak{M}_{1}$. Since $I / J \notin Q$ there must exist some $\alpha$ such that $I / J \in \mathscr{M}_{\alpha}$ but $I / J \notin \mathfrak{T}_{\alpha+1}$. If $\nu$ is the largest ordinal for which $\mathfrak{M}_{\gamma}$ contains a nonzero homomorphic image of $I$, then all $0 \neq I / J \notin \Re_{\gamma+1}$. But $R \in \Re_{\gamma+2}$ so that every nonzero ideal of $R$, and in particular $I$, has a nonzero 
image in $\mathfrak{T}_{\gamma+1}$. This contradiction shows that $Q$ has property (B) and hence is in $y$.

Finally, let $\mathfrak{H} \in \mathcal{Y}$ with $\mathfrak{H} \subseteq \mathscr{T}$. Then $\mathcal{H C}$ has property (C) so $\mathfrak{H C} \subseteq \mathbb{M}_{1}$. Assume for induction that $\mathfrak{H} \subseteq \mathfrak{T}_{\alpha}$ for all $\alpha<\beta$, then clearly $\mathfrak{H} \subseteq \mathbb{M}_{\beta}$ when $\beta$ is a limit ordinal. Thus suppose $\mathfrak{H} \subseteq \mathfrak{M}_{\beta-1}$, then if $R \in \mathcal{F}$ by (B) every $0 \neq I \triangleleft R$ has some $0 \neq I / J \in \mathcal{H} \subseteq \mathscr{M}_{\beta-1}$. Thus by definition $R \in \mathbb{M}_{\beta}$, so by induction $R \in \mathscr{N}_{\beta}$ for all $\beta$, whence $\mathfrak{H} \subseteq Q$.

Note that we can construct a class containing no maximal semisimple class, using a countably infinite subset $\left\{R_{i}\right\}(i=1,2, \cdots)$ of the set $V$ of Example 2. Let $F \mathscr{M}$ denote the smallest hereditary semisimple class [2] containing $\mathfrak{T}$. Set $\mathfrak{T}_{1}=F\left\{R_{1}\right\}$ and for $n \geqq 1$ define $\mathfrak{M}_{n+1}=F\left(\mathfrak{M}_{n} \cup\left\{R_{n+1}\right\}\right)$. Then let $\mathfrak{N}=\bigcup_{n} \mathfrak{M}_{n}$, and suppose $Q \subseteq \mathfrak{N}$ is semisimple. If there were no $n$ such that $Q \subseteq \mathbb{N}_{n}$ then we could find an infinite ascending sequence of integers $\left\{n_{i}\right\}$ such that for some $K_{i} \in Q$ we would have $K_{i} \in \mathfrak{M}_{n_{i}}, K_{i} \notin \mathfrak{M}_{n_{i-1}}$. But $K=\sum \oplus K_{i} \in \mathcal{Q}$ so $K \in \mathfrak{M}_{n}$ for some $n$. Thus since $\mathfrak{M}_{n}$ is hereditary we would have all $K_{i} \in \mathscr{T}_{n}$. From this contradiction we conclude that $\Theta \subseteq \mathfrak{M}_{n}$ for some $n$. Since a simple ring is in $F \mathscr{T}$ if and only if it is in $\mathscr{T}$ (when $\mathscr{T}$ is hereditary) it follows that $\mathfrak{M T}_{n}$ (and hence $\mathcal{O}$ ) is not maximal. Also note that in Example 1 the class $\mathscr{N}=\delta \mathcal{U}\{J\} \cup\{K\}$ does not have property (C) but does contain a largest class $\delta u\{J\}$ with property (C).

We will use the constructions of Theorems 3 and 4 to establish "largest" theorems dual to the smallest theorems of $\$ \S 2$ and 3.

Theorem 5. Let $F: J \rightarrow J$ be s-admissible, then any class $\mathfrak{T}$ containing a largest class $\mathbb{N}_{1}$ with property (A) contains a largest $\bar{\rho} \in \mathbb{R}$ such that $F S \bar{P}=S \bar{P}$.

Proof. By Theorem 3, I contains a largest $\beta \in R$. By Theorem 2, there exists a smallest $\overline{\mathscr{N}} \in \mathcal{Y}$ such that $\delta \mathscr{P} \subseteq \overline{\mathscr{N}}$ and $F \overline{\mathscr{N}}=\overline{\mathscr{N}}$. Thus $\bar{\rho}=\mathcal{u \mathscr { T }} \subseteq \mathcal{U} \mathcal{P}=\mathcal{P} \subseteq \mathscr{T}$. If $\mathfrak{H} \subseteq \mathscr{N}$ is a radical class then $\mathfrak{H C} \subseteq \mathcal{P}$ and so

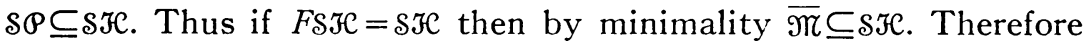
$\mathfrak{H}=\mathcal{U} \mathfrak{C} \subseteq \mathcal{U \mathscr { N }}=\bar{\rho}$ and hence $\bar{\rho}$ is the largest subclass of $\mathfrak{T C}$ for which $F \delta \overline{\bar{\rho}}=s \bar{\rho}$.

Note that for Theorem 5 the function $F$ only needs to be defined for the semisimple subclasses of $w$. Note that a property $p$ of radical classes defines a subclass of $R$, namely the class of all $P \in R$ with property $p$. Conversely any $V \subseteq R$ defines a property of radical classes, and similarly any $\mathfrak{X} \subseteq \mathcal{Y}$ defines a property of semisimple classes. Let $V \subseteq R$ and $x \subseteq \mathcal{Y}$ be called dual if $\rho \in \mathcal{V}$ if and only if $s \rho \in x$. Note that $R$ and $\mathcal{Y}$ are themselves dual.

Corollary 3. Let $v \subseteq R$ and $x \subseteq \mathcal{Y}$ be dual. Then every class $\mathfrak{T}$ 
containing a largest class $\mathfrak{T}_{1}$ with property (A) contains a largest class $\odot \in \mathcal{V}$ if and only if every $\Re \in \mathcal{Y}$ is contained in a smallest $Q \in X$.

Proof. The sufficiency is proved in precisely the way of the proof of Theorem 5, replacing " $F \overline{\mathscr{N}}=\overline{\mathscr{N}}$ " with " $\bar{\Re} \in X$." Thus let $\Re \in \mathcal{Y}$. Then $\mathcal{U} \mathfrak{X}$ is a radical class, so itself has property (A). Thus if $\mathcal{P} \subseteq \mathcal{U} \mathscr{Y}$ is a largest subclass with $\rho \in \mathcal{V}$ it clearly follows that $\Re \subseteq \delta \rho$ where $s \odot \in X$ and is the smallest member of $X$ containing $\Re$.

As an application we have from [2]:

CoROllary 4. Any class $\mathfrak{T}$ containing a largest class $\mathfrak{T C}_{1}$ with property (A) contains a largest radical class with hereditary semisimple class.

The corresponding duality theorem for semisimple classes is proved in a similar way and will be stated without proof:

THEOREM 6. Let $F: R \rightarrow J$ be admissible, then any class $\mathbb{M}$ containing a largest class $\mathfrak{M}_{1}$ with property $(\mathrm{C})$ contains a largest $\Theta \in \mathcal{Y}$ such that $F น \mathcal{Q}=$ UQ .

Corollary 5. Let $U \subseteq R$ and $X \subseteq Y$ be dual. Then every class $\mathfrak{T}$ containing a largest class $\mathfrak{M}_{1}$ with property $(\mathrm{C})$ contains a largest class

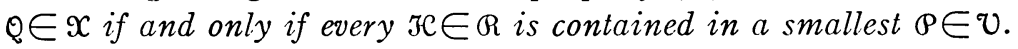

From this theorem it clearly follows that such a class contains a largest semisimple subclass whose radical is hereditary [6] or strongly hereditary [1].

The dualization may also be taken relative to a specific class:

Corollary 6. Let $v \subseteq R$ and $x \subseteq \mathcal{Y}$ be dual. Let $\mathfrak{T}$ have a largest subclass with property (A) and let $\odot$ be the largest radical subclass of $\Re$. Then $\mathfrak{T}$ has a largest $\bar{P} \in \mathcal{V}$ if and only if $S \odot$ is contained in a smallest $Q \in X$. Similarly, if $\mathfrak{T}$ has a largest subclass satisfying (C) and largest semisimple class $\mathcal{Q}$, then $\mathfrak{T}$ contains a largest $\overline{\mathcal{Q}} \in X$ if and only if $U \mathcal{Q}$ is contained in a smallest $P \in \mathcal{V}$.

Note that the condition that $\mathfrak{M}$ have a largest subclass with property (A) (or property (C)) is sufficient for the theorems of this section. It is not known whether or not the condition is also necessary, but it is expected that in general it will not be.

\section{REFERENCES}

1. W. G. Leavitt, Strongly hereditary radicals, Proc. Amer. Math. Soc. 21(1969), 703-705.

2. - Hereditary semisimple classes, Glasgow Math. J. (to appear). 
3. Ju. M. Rjabuhin, On imbeddings of radicals, Bul. Akad. Stiince RSS Moldoven. 1963, no. 11, 34-41. (Russian) MR 34 \#7572.

4. N. J. Divinsky, Rings and radicals, Univ. of Toronto Press, Toronto, Ont., 1965. MR 33 \#5654.

5. W. G. Leavitt and E. P. Armendariz, Nonhereditary semisimple classes, Proc. Amer. Math. Soc. 18(1967), 1114-1117. MR 36 \#3838.

6. A. E. Hoffman and W. G. Leavitt, Properties inherited by the lower radical, Portugal. Math. (to appear).

7. Ju. M. Rjabuhin, Lower radicals of rings, Mat. Zametki 2(1967), 239-244. (Russian) MR 36 \#220.

UNIVERSity OF Nebraska, LinCOLN 